\title{
Current Research on Telemonitoring In Patients with Diabetes Mellitus: A Short Pragmatic Narrative Review
}

Andrès Emmanuel $1,2 *$, Meyer Laurent ${ }^{3}$, Zulfiqar AA ${ }^{2,4}$, Hajjam Mohamed ${ }^{5}$, Talha Samy ${ }^{2,6}$, Ervé Sylvie ${ }^{7}$, Hajjam Jawad ${ }^{7}$, Bahougne Thibault ${ }^{3}$, Doucet Jean ${ }^{4}$, Jeandidier Nathalie ${ }^{3}$, and Hajjam El Hassani Amir ${ }^{8}$

${ }^{1}$ Department of Internal Medicine, Diabetes and Metabolic Diseases of Medical Clinic B, France

${ }^{2}$ Faculty of Medicine of Strasbourg, France

${ }^{3}$ Department of Endocrinology and Diabetology of Medical Clinic B, France

${ }^{4}$ Department of Internal Medicine, Geriatrics and Therapeutics, France

${ }^{5}$ Predimed Technology, France

${ }^{6}$ Department of Physiology and Functional Explorations, France

${ }^{7}$ Center of Expertise for Information and Communication Technologies for Autonomy (CENTICH), France

${ }^{8}$ Research team EA 4662 Nanomedicine, Imaging \& Therapeutics, France

*Corresponding author: Emmanuel Andrès, Department of Internal Medicine, Diabetes and Metabolic Diseases of Medical Clinic B, Strasbourg University Hospitals, 1, Hospital gate, 67091 Strasbourg cedex France

submission: November 27, 2018; Published: January 03, 2019

\begin{abstract}
Background: This is a narrative review of remote monitoring (telemedicine) projects within the field of type 1 and type 2 diabetes, with special attention placed on telemedicine 2.0 projects and studies.

Material and method: A literature search were performed using the PubMed database of US National Library of Medicine, along with Scholar Google. Textbooks on telemedicine and e-Health, from the American Diabetes Association (ADA) and the European Association for Study the Diabetes (EASD), as well as information from international meetings and commercial sites on the Web were used.

Result: Since the beginning of the 1990's, several telemedicine projects and studies focused on type 1 and type 2 diabetes have been developed. Mainly, these projects and studies show that telemonitoring diabetic result in: improved blood glucose control; a significant reduction in HbA1c; improved patient ownership of the disease; greater patient adherence to therapeutic and hygiene-dietary measures; positive impact on co-morbidities (hypertension, weight, dyslipidemia); improved quality of life for patients; and at least good patient receptivity and accountability. To date, the magnitude of its effects remains debatable, especially with the variation in patients' characteristics (e.g. background, ability for self-management, medical condition), samples selection and approach for treatment of control groups. Over the last 5years, numerous telemedicine projects based on connected objects and new information and communication technologies (ICT) (elements defining telemedicine 2.0) have emerged or are still under development. Two examples are the DIABETe and Telesage telemonitoring project which perfectly fits within the telemedicine 2.0 framework, being the firsts to include artificial intelligence with MyPrediTM and DiabeoTM (AI).
\end{abstract}

Keywords: Telemedicine; Telemonitoring; Artificial intelligence; Information and communication technology; Web; Diabetes; Heart failure; Chronic disease

\section{Introduction}

Intensive glucose control has been shown to delay or prevent the development of micro- and macrovascular complications related to diabetes. However, it is estimated that 43.2 to $55.6 \%$ of adults with type 2 diabetes do not meet the reference target for glycemic control (hemoglobin A1c $[\mathrm{HbA} 1 \mathrm{c}]<7.0 \%$ ). Factors that may contribute to sub-optimal blood glucose (BG) control include inadequate home BG monitoring, non-adherence or non-compliance with medications or lifestyle changes (nutrition and sport), suboptimal patient education about the disease, and limited access to health professionals [1-3]. In the absence of timely and accurate data on home BG values, healthcare professionals may be reluctant, and rightly so, to aggressively intensify oral hypoglycemic agents or insulin treatments for fear of hypoglycemia. In this setting, 50\% of diabetic patients, especially elderly patients followed in hospital or in referral centres, have macrovascular complications as myocardial infarction (MI), obliterating arteritis of the lower limbs or stroke, even chronic heart failure (CHF) in the elderlies [4]. These patients have a high mortality rate; with $20 \%$ of deaths occur within $5 y e a r s$ after the first cardiovascular event. In this context, patients are often hospitalized, with prolonged and iterative hospitalization [2]. 
In practice, the main causes of diabetes deterioration required medical intervention are related to: the diabetes, with non-therapeutic adherence and compliance, poor nutrition, poor adherence to prescribed lifestyle changes and therapy; the decompensation of diabetic comorbidities and macrovascular complication; and community-based infections. In this context, telemedicine may be an effective approach to solving problems of education, compliance, and monitoring and provider access $[2,5]$. BG control could be safely improved by basing drug changes on home BG readings and transmitting them in near-real time to providers. In this setting, telemedicine may also be an effective solution to monitor the complications of the diabetes, especially macrovascular complications (e.g. MI, HF, etc.) and comorbidities (e.g. arterial hypertension).

In this article, we review with a pragmatic mind and a clinical vision the literature in the field of remote monitoring (telemonitoring) of diabetic patients.

\section{First-Generation Telemedicine Projects and Studies in the Field of Diabetes}

Since the early 1990 s to the end of 2010 , numerous telemedicine projects and studies have been developed in the field of diabetes [6-27]. Practically all of them have investigated "telemonitoring" or "telephone follow-up" (defined terms in Table 1), especially to monitor BG levels. For the majority of them, they were conducted on specific population of poor controlled type 1 and type 2 diabetic patients, as: children and young people $(n=3)$, elderly patients $(\mathrm{n}=2)$, patients with intensified therapy $(\mathrm{n}=2)$, patients under insulin pump therapy $(\mathrm{n}=1)$; and patients with complicated or complex diabetes $(n=2)$.

To our knowledge, to date, no project has been published on "tele-consultation" and "tele-expertise" (defined terms in Table 1) in the area of diabetes domain, as defined under European or French legislation [28]. Several of such projects have been developed but no but no formal scientific conclusions are currently available about the usefulness of these telemedicine technologies [29].

It is worth bearing in mind that these projects and studies, particularly the earlier ones, more closely resembled as a telephone follow-up with care providers (such as a nurse) traveling to the diabetic patient's home, rather than telemedicine use as we think of it nowadays with nonintrusive, automated, smart telemonitoring using employing remote sensors via modern communication technology (e.g. smartphone) or even artificial intelligence (AI) (Table 1). Thus, they characterize in our opinion "first-generation" telemedicine projects and studies.

Table 1: Glossary of terms and definitions in the field of telemedicine [29].

\begin{tabular}{|c|c|}
\hline Telemedicine & Provision of remote patient care and consultation using telecommunication technologies. \\
\hline Telemonitoring & $\begin{array}{l}\text { This telemedicine practice allows a healthcare professional to remotely interpret the data necessary for the patient's } \\
\text { medical follow-up in order to make decisions about his/her care. Remote data collection from a patient through a } \\
\text { connected device or questionnaires to monitor his/her vital parameters and symptoms at home on a daily basis. }\end{array}$ \\
\hline Teleexpertise & $\begin{array}{c}\text { This practice of telemedicine consists, for a medical professional, to seek the opinion of one or more medical profes- } \\
\text { sional experts regarding elements of the patient's medical file. Remote seeking by a health professional of a second } \\
\text { medical opinion via sending of images (scanner, X-ray, eye fundus, etc.) and sometimes exchange by Internet-based } \\
\text { videoconference }\end{array}$ \\
\hline Teleconsultation & $\begin{array}{l}\text { This telemedicine practice allows a medical professional to hold a consultation with a patient remotely. In the context } \\
\text { of a teleconsultation, the patient can have at his/her side a health professional assisting the remote professional as well } \\
\text { as a psychologist. Second opinion consultation by specialist }\end{array}$ \\
\hline Telemedicine 2.0 & $\begin{array}{l}\text { Over the last decade, the Internet has become increasingly popular and is now an important part of our daily life. The } \\
\text { use of "Web 2.0" technologies in health/medicine care or in telemedicine is referred to as "Health } 2.0 \text { " or "Medicine } \\
\text { 2.0", and "telemedicine 2.0" }\end{array}$ \\
\hline Artificial intelligence & $\begin{array}{l}\text { This concept makes it possible for machines to learn from experience, adjust to new inputs and perform human-like } \\
\text { tasks. These processes include learning (the acquisition of information and rules for using the information), reasoning } \\
\text { (using the rules to reach approximate or definite conclusions) and self-correction. Particular applications of AI include } \\
\text { expert systems, speech recognition, and machine vision }\end{array}$ \\
\hline
\end{tabular}

Using PubMed data-base and Google scholar, we have identified more than 20 reports of first-generation telemonitoring studies in the field of diabetes, including type 1 and type 2 diabetic patients, involving the upload and direct transmission of blood-glucose data by diabetic patients to providers via cellular telephone, telephone land line, or a Web-based program. The results of these studies were mixed, perhaps because many studies did not target diabetic patients with poor baseline BG control, or the interval between glucose transmission and follow-up was delayed or unspecified, or mainly with no therapeutic intervention (therapeutic inertia). None of these reports evaluated the intensity of intervention required to sustain achieved reductions in $\mathrm{HbA1c}$ after the implementation of home telemonitoring.

As with $\mathrm{CHF}$, the results of these first-generation telemedicine projects differed from study to study, with fairly inconclusive results as to their potential clinical benefits in terms of balancing diabetes and the associated-metabolic problems, re-hospitalization and decreased morbidity or mortality, particularly regarding the statistical significance of the results $[29,30]$. As a consequence, experts have share now widely divergent opinions on the actual utility of telemedicine in diabetic patient management. 
To our knowledge, it should be emphasized that the firstgeneration studies and trials on telemedicine in diabetic patients were at times conducted with:

A. Inappropriate methodologies, involving unsuitable patient groups, (such as well-balanced diabetic patients, diabetic patients without any complication), of small-sized patient samples, and with very short follow-up periods (between 3 months to 1 year);

B. Not well-structured follow-up organization, with nonnot specialized staff to alarms, or without any association of patients' general practitioners, specialists of diabetes management or endocrinologists and nor any optimized management process or algorithm;

C. Several alarms arising too late, without therapeutic response (no specified therapeutic protocol available);

D. No associated educational programs;

E. Absence of a human interface or contact between the telemedicine solution and the patients.

Moreover, most of these studies were only based on glycemic control, without including other warning or monitoring parameters related to comorbidities or diabetic complication (e.g. tensiometer heartrate, balance), with an under-utilization of the deployed device. Thus, in our opinion, these facts explain that the demonstration of any benefit with these first-generation studies was "illusory", in particular in terms of statistical significance!

Besides these medical considerations, it is worth noting that an economical aspect must be investigated and consolidated in future telemedicine projects to promote the development of telemedicine in diabetes and legitimize it, especially in regard of the budgetary constraints affecting insurance and mutual health insurance companies. Things are less advanced than in the field of chronic heart failure telemonitoring. To our knowledge, only Biermann's study is dedicated to this theme of economical aspect [11]

To date, none of the learned societies (e.g. American Diabetes Association [ADA], European Society of Diabetes [ESD]) involved in the topic of diabetes has, to our knowledge, made any formal recommendation as to whether or not telemedicine is of benefit to type 1 or type 2 diabetic patients. This is not the case in the setting of $\mathrm{CHF}$, where factual data and medico-economic studies are more numerous, better documented and consolidated (more mature field). In fact, the 2016 European Society of Cardiology (ESC) guidelines for the diagnosis and treatment of acute and chronic heart failure have recommended telemonitoring of heart failure patients with a recommendation Grade of recommendation IIb, and level of evidence B [31].

\section{Second-Generation Telemedicine Projects and Studies} in the Field of Diabetes

Over the last ten years, "second-generation" telemedicine projects and studies have been developed in the setting of diabetes management, especially in the setting of telemonitoring [32-
38], as defined in Table 1. These projects and studies have for main objectives to evaluate the use of technology to implement medical and cost-effective health care management on a large scale for diabetes management. Compared to the aforementioned projects, most of the second-generation projects related to diabetes telemonitoring (for type 1 diabetics patients: $n=1$; for type 2 : $n=5$ ) incorporate the following:

A. Self-administered medical questionnaires or forms on: symptoms, signs of diabetes decompensation; BG levels;

B. Tools for medical education, particularly disease selfappropriation, food hygiene, and physical activity;

C. Tools for patient motivation;

D. Tools for therapeutic and hygiene observance;

E. Tool to remote comorbidities (e.g. arterial hypertension, obesity, dyslipidemia);

F. Tools for interaction between the patient and healthcare professionals like telephone support centers, tablets, and Websites.

\section{The DiaTel study}

The DiaTel study compared the short-term efficacy of home telemonitoring coupled with active medication management by a nurse practitioner with a monthly care coordination telephone call on glycemic control in veterans with type 2 diabetes. The included patients were taking oral hypoglycemic agents and/or insulin for $\geq 1$ year and had HbA1c $\geq 7.5 \%$ ).

At enrollment, the patients were randomly assigned to either: active care management (AMC) with home telemonitoring (HT) (ACM+HT group, n=73); and a monthly care coordination telephone call (CC group, $\mathrm{n}=77$ ). Both groups received monthly calls for DM education and self-management review. ACM+HT group participants transmitted BG, blood pressure (BP), and weight to a nurse practitioner; the nurse practitioner adjusted medications for glucose, BP, and lipid control based on established ADA targets.

Baseline characteristics of the patients in the DiaTel study were similar in both groups, with mean HbA1c: $9.4 \%$ in the CC group vs. $9.6 \%$ in ACM+HT group [32,33]. Compared with the CC group, the ACM+HT group demonstrated significantly larger decreases in HbA1c (principal criterion) at 3months $(1.7 \%$ vs. $0.7 \%)$ and 6 months ( $1.7 \%$ vs. $0.8 \%$; $p<0.001$ for each), with most improvement occurring by 3 months.

\section{The utah remote monitoring project}

The Utah Remote Monitoring Project was a non-randomized prospective observational pre- and post-intervention study [34]. The included patients were patients with uncontrolled type 2 diabetes and/or arterial hypertension. They have been enrolled from four rural and two urban primary care clinics and one urban stroke center participated in a telemonitoring program $(n=109)$. The primary clinical outcome measures were changes in HbA1c and BP. Other outcomes included fasting lipids, weight, patient 
engagement, diabetes knowledge, arterial hypertension knowledge, medication adherence, and patient perceptions of the usefulness of the telemonitoring program.

The patients were randomized on two groups on telemonitoring delivery methods. The first was a remote monitoring device for BP and heart rate. Patients used their own glucose meters to measure BG and were provided with an electronic digital scale to measure their weight. The device was programmed to sound an alarm at a pre-specified patient-referred time to prompt the patient to initiate a telemonitoring session. Patients were asked to enter data several times during the week. The device was programmed to ask how patients were feeling that day and whether they had taken their medications and then receive a prompt to take the measures. After, the patient received a series of education messages, focused on teaching patients about their diseases (diabetes, arterial hypertension) and associated comorbidities. The second telemonitoring delivery method use of an interactive voice response (IVR) system. Patients were provided with a BP monitor and electronic digital scales, but they used their own BG meter. The patients have to use the same process described above but received a call from the telemonitoring IVR service at a prespecified. Medical providers were contacted either via a note in the electronic medical record (or immediately if there was a concern, in person or by telephone) if there was an out-of-range value (decided by individual providers or clinics as a value that was high or low).

In this study, the mean HbA1c (principal criterion) decreased: $9.73 \%$ at baseline vs. $7.81 \%$ at the end of the program $(\mathrm{p}<0.0001)$. Systolic BP (principal criterion) also declined significantly: $130.7 \mathrm{mmHg}$ at baseline vs. $122.9 \mathrm{mmHg}$ at the end $(\mathrm{p}=0.0001)$. Low-density lipoprotein content decreased significantly: $103.9 \mathrm{mg} /$ $\mathrm{dL}$ at baseline vs. $93.7 \mathrm{mg} / \mathrm{dL}$ at the end $(\mathrm{p}=0.0263)$. Knowledge of diabetes and arterial hypertension increased significantly $(p<0.001$ for both). Patient engagement and medication adherence also improved, but not significantly. Per questionnaires at study end, patients felt the telemonitoring program was useful.

\section{Randomized trial on home telemonitoring for the man- agement of metabolic and cardiovascular risk in pa- tients with type 2 diabetes}

This study evaluated whether a home telehealth (HT) system can improve metabolic control and overall cardiovascular risk in individuals with type 2 diabetes, compared with usual practice. This study was a randomized, parallel-group, open-label, multicenter study conducted in general practice (29 general practitioners) including 302 patients, with a follow-up of 12 months.

The HT system (for the telemedicine group of diabetic patients, $\mathrm{n}=153$ ) offers to the patient the possibility to monitor body weight, BG values, and BP values, associated with remote educational support and feedback to the general practitioner.

Use of the HT system was associated with a statistically significant reduction in $\mathrm{HbA1}$ c levels (principal criterion) compared with the control group: estimated mean difference of $0.33 \pm 0.1$ $(p=0.001)$. No difference was documented for body weight, BP, and lipid profile (all principal criteria). The proportion of patients reaching the target of $\mathrm{HbA1c}(\mathrm{HbA} 1 \mathrm{c}<7.0 \%)$ was higher in the HT group than in the control group after 6 months: $33.0 \%$ vs. $18.7 \%$ ( $p=0.009$ ) and 12 months: $28.1 \%$ vs. $18.5 \%(p=0.07)$. As for quality of life (evaluated with the 36-item Short Form health survey), significant differences in favor of the HT group were detected as for physical functioning $(\mathrm{p}=0.01)$ and mental health $(\mathrm{p}=0.005)$. On an economic level, a lower number of specialist visits was reported in the telemedicine group: incidence rate ratio of 0.72 (95\% confidence interval, 0.51-1.01; $\mathrm{p}=0.06$ ).

\section{Study assessed the utility and cost-effectiveness of an automated diabetes remote monitoring and manage- ment system (DRMS)}

This study assessed the utility and cost-effectiveness of an automated Diabetes Remote Monitoring and Management System (DRMS) in glycemic control versus usual care. In this randomized, controlled study, patients with uncontrolled diabetes on insulin were randomized to use of the DRMS or usual care.

Participants in both groups were followed up for 6 months and had three clinic visits during the study period (at 0,3 , and 6 months. The DRMS used text messages or phone calls to remind patients to test their BG and to report results via an automated system, with no human interaction unless a patient had severely high or low BG. The DRMS made adjustments to insulin dose(s) based on validated algorithms. Participants reported medication adherence through the Morisky Medication Adherence Scale-8, and diabetes-specific quality of life through the diabetes Daily Quality of Life questionnaire. A cost-effectiveness analysis was conducted based on the estimated overall costs of DRMS and usual care.

A total of 98 diabetic patients (60\% of female) treated with insulin therapy were enrolled. The mean age of the patients was 59years. At the end, 87 patients (89\%) have completed the followup. HbA1c was similar between the DRMS and control groups at 3 months: $7.60 \%$ vs. $8.10 \%$ and at 6 months: $8.10 \%$ vs. $7.90 \%$ (p=ns) (principal criterion) [39]. Changes from baseline to 6 months were not statistically significant for self-reported medication adherence and diabetes-specific quality of life, except for the Daily Quality of Life-Social/Vocational Concerns subscale score $(p=0.04)$.

\section{The telescot diabetes pragmatic multicenter randomized controlled trial}

The Telescot Diabetes is a randomized, parallel, investigatorblind controlled trial with centralized randomization in family practices in four regions of the United Kingdom [40]. This study included 321 patients with relatively well-controlled type 2 diabetes, with an $\mathrm{HbA} 1 \mathrm{c}>7.46 \%$.

In Telescot Diabetes, 160 people were randomized to the intervention group and 161 to the usual care group. The supported telemonitoring intervention involved self-measurement and transmission to a secure website of twice-weekly morning and evening glucose for review by family practice clinicians who were not blinded to allocation group. The control group received usual care, with at least annual review and more frequent reviews for 
people with poor glycemic or BP control. HbA1c assessed at $9^{\text {th }}$ month was the primary outcome.

The mean (SD) HbA1c at follow-up was 7.92\% in the intervention group vs. $8.36 \%$ in the usual care group. For primary analysis, adjusted mean HbA1c was $0.51 \%$ lower $95 \%$ CI $0.22 \%$ to $0.81 \%$, (principal criterion) ( $\mathrm{p}=0 \cdot 0007$ ). For secondary analyses, adjusted mean ambulatory systolic BP was $3.06 \mathrm{mmHg}$ lower $(95 \%$ CI 0.56-5.56 mmHg, $\mathrm{p}=0.017$ ) and mean ambulatory diastolic BP was $2.17 \mathrm{mmHg}$ lower (95\% CI 0.62-3.72, p=0.006) among people in the intervention group when compared with usual care after adjustment. No significant differences were identified between groups in weight, treatment pattern, adherence to medication, or quality of life in secondary analyses. During the study, the number of telephone calls was greater between nurses and patients in the intervention compared with control group: rate ratio of 7.50 (95\% CI 4.45-12.65, p<0.0001) but no other significant differences between groups in use of health services were identified between groups.

\section{Educ@dom}

Educ@dom is a multicenter, randomized, controlled, prospective study. The primary objective of this study is to compare the efficacy of telemonitoring to standard monitoring in terms of changes in HbA1c after a 1-year follow-up period. The secondary objectives are clinical (changes in knowledge, physical activity, weight, etc.) and medical-economic.

The Educ@dom study included 282 patients, 141 patients in each arm. For patients in the intervention group, the device will be given to them for 1 year and then withdrawn during the second year of follow-up. The anticipated benefits of this research are an improvement in BG management in patients with type 2 diabetes by improving their lifestyle whilst rationalizing recourse to consultations in order to reduce the incidence of complications and cost in the long term. The results of this study are expected in 2019-2020

\section{New Generation Projects and Studies in Diabetes}

Over the last 5 years, new-generation telemedicine projects and studies have emerged in the setting of chronic diseases setting, especially in the setting of chronic heart failure, chronic obstructive pulmonary diseases and type 1 and type 2 diabetes [41,42]. They support transmission and remote interpretation of patients' data for follow-up and preventive interventions. These projects and studies have for main objectives to evaluate the use of technology to implement medical and cost-effective health care management on a large scale for diabetes management. Using PubMed database and Google scholar we have identified three of such projects and studies in the field of diabetes management: Telemonitoring and Health Counseling for Self-Management Support from Lindberg et al., TELESAGE, DIABETe. Of note for the first time, one the telemedicine projects developed for chronic diseases management, the TIMHF2 study [43], has recently demonstrated the usefulness of telemedicine in chronic heart failure, with statistically significance, in a prospective randomized study (the "gold-standard" of Evidence Based Medicine [EBM]).

Between August 13, 2013, and May 12, 2017, 1,571 patients (mean age of 70years) were included in the TIM-HF2 study and randomly assigned to remote patient management $(n=796)$ or standard care $(n=775)$ [44]. At baseline, all patients exhibited a left ventricular ejection fraction of $<45 \%$ and NYHA II or III, while receiving treatment with diuretics. In TIM-HF2 study, the percentage of days lost due to unplanned cardiovascular hospital admissions and all-cause death was: 4.88\% (95\% CI 4.55-5.23) in the remote patient management group vs. $6.64 \%(6.19-7.13)$ in the standard care group (ratio 0.80, 95\%CI: 0.65-1) ( $\mathrm{p}=0.0460$ ). The all-cause death rate was: 7.86 (95\% CI: 6.14-10.10) per 100 person-years of follow-up in the remote patient management group vs. 11.34 (95\% CI: 9.21-13.95) per 100 person-years of follow-up in the standard care group (hazard ratio [HR] 0.70, 95\%CI: $0.5-$ 0.96) ( $p=0.0280$ ) (Figure 1) [43]. Cardiovascular mortality did not significantly differ between both groups (HR 0.671, 95\%CI: 0.45 1.01; $\mathrm{p}=0.056$ ).

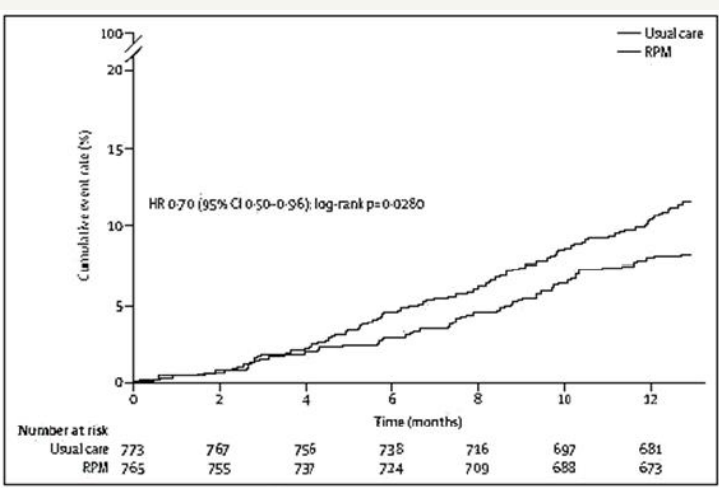

Figure 1: TIM-HF2 trial. Rate of cumulative events in patients randomly assigned to remote patient management $(n=796)$ or usual care $(\mathrm{n}=775)$ (adapted from [43])

The TIM-HF2 study utilized a noninvasive, multi-parameter telemonitoring system installed in the patient's home, comprising a three-channel ECG, BP-monitoring device, and weighing scales, by means of which the information was transferred remotely [45]. Patients received a mobile phone in order to contact the telemedical center in case of emergency. Patients were likewise followed via monthly phone interviews. For this TIM-HF2 care strategy, the key component was a well-structured telemedical center with physicians and HF nurses ("center of coordination") available 24 hours a day and every day a week, able to act promptly according to the individual patient risk profile. The actions taken by the telemedical center staff included changes in medication and admission to hospital, as needed, in addition to educational activities.

In this setting, we believe that, thanks to technological innovations in connected health-monitoring devices, the telemonitoring of type 2 diabetic patients using therapeutic educational tools is likely to help them adapt their treatment and lifestyle habits, and therefore improve BG management. 
These new generation telemedicine projects in diabetes (Telemonitoring and Health Counseling for Self-Management Support from Lindberg et al., TELESAGE, DIABETe) are often known as "telemedicine 2.0" projects, given that they all utilize new Information and Communication Technologies (ICT) and the Web (tools for the "e-Health 2.0") (as defined in Table 1).

Most projects and studies rely on the standard connected tools for monitoring type 1 and type 2 diabetes, such as glucose meters, $\mathrm{BP}$, heart rate monitors, weighing scales, and pulse oximeters, which relay the collected information via Bluetooth, 3G or 4G. Several projects also include continuous glycemic monitoring solution and often a video-call. Several of these telemedicine projects use machine learning, also called artificial intelligence (AI), in order to be able to:

Adjust the BG level to the patient's activity (software DiabeoTM [see below]);

A. Predict patient risks of diabetes decompensation. In this later situation, the cloud-based software aggregates, cleans, and analyzes patient data to allow for identifying patterns that may indicate potential risks and provide predictive insights on healthcare outcomes, as the software MyPrediTM (see below).

In the setting of chronic diseases, as in chronic heart disease or in diabetes, several informatics solutions or tools have been developed and used, such as Artificial Neural Networks (ANN) algorithms, data mining software, ontology [46-48]. In this context of $\mathrm{AI}$, three clinical datasets are of particular interest:

\section{A. patients' phenotype;}

B. patients' electronic medical records containing physicians' notes, laboratory test results, as well as other information on diseases, treatments, and epidemiology that may be of interest for association studies and predictive modeling on prognosis and drug responses; and

C. literature knowledge including rules on diabetes management.

Besides these tools, it must be emphasized that diabetes telemonitoring may use, as for CHF telemonitoring, implantable invasive devices that send either sporadically or continuously data to the receiving physician (automatic telemonitoring) (outside the scoop of this paper). In management of diabetes, implantable telemonitoring devices for multi-parameters including mainly BGinsulin levels monitoring have recently proven to be an effective approach.

\section{Telemonitoring and health counseling for self- management support of patients with type 2 diabetes}

The objective of this study (Telemonitoring and Health Counseling for Self-Management Support) was to investigate whether the introduction of a health technology-supported self-management program involving telemonitoring and health counseling had beneficial effects on HbA1c, other clinical variables (weight, body mass index, BP, blood lipid profile), and health-related quality of life (HRQoL), as measured using the Short Form Health Survey (SF-36) version 2 in patients with type 2 diabetes (Figure 2) [45].

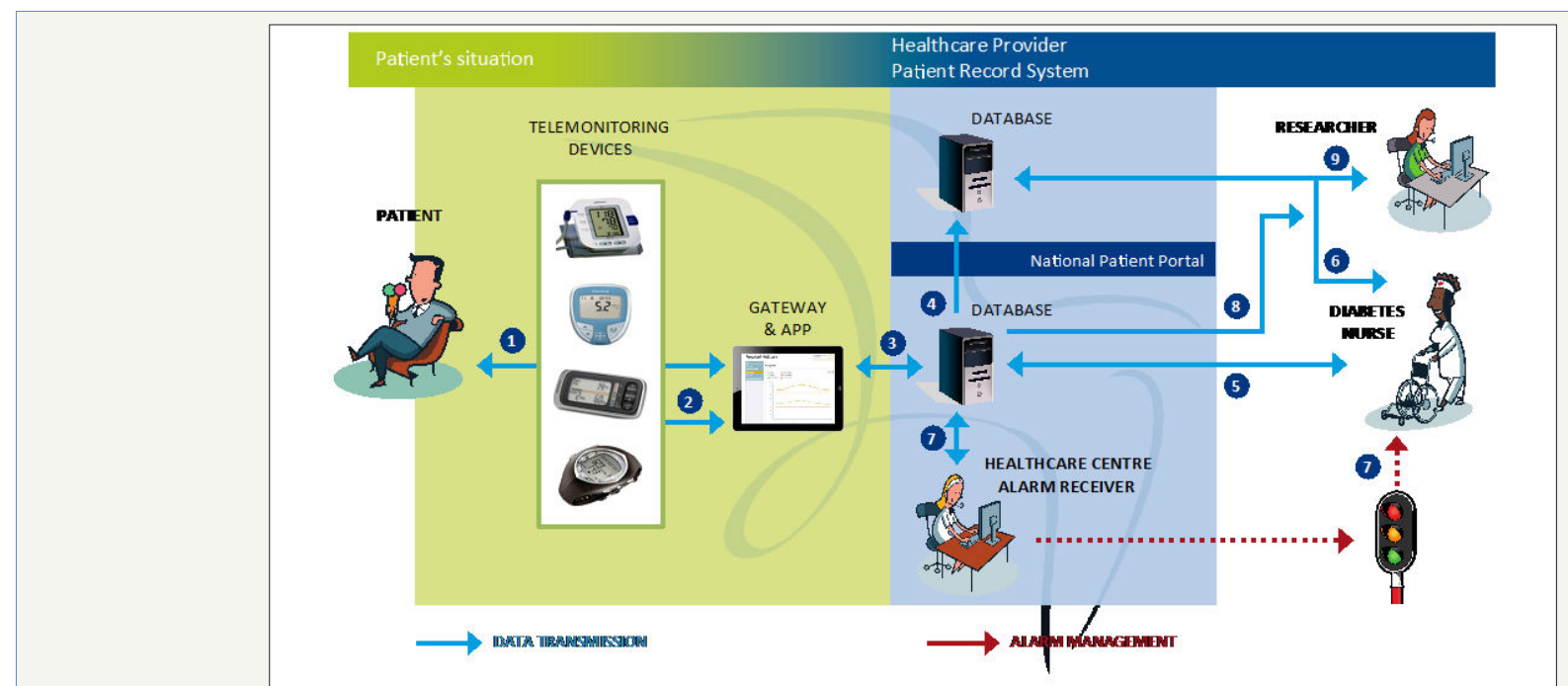

Figure 2: Telemonitoring devices and information flow during the field trial (adapted from [45]).

This was a pragmatic randomized controlled trial of patients with type 2 diabetes. Both the control $(n=79)$ and intervention groups $(n=87)$ received usual care. The intervention group also participated in additional health promotion activities with the use of the Prescribed Healthcare Web application for self-monitoring of BG and BP. About every second month or when needed, the general practitioner or the DM nurse reviewed the results and the health care activity plan.
Analyze of the data showed that there were no significant differences between the groups in the primary outcome HbA1c level ( $p=0.33$ ), and in the secondary outcome HRQoL as measured using SF-36. A total of $80 \%$ of the patients in the intervention group at the baseline, and $98 \%$ of the responders after 19-month intervention were familiar with using a personal computer $(p=0.001)$. After 19 months, no responders reported significantly poorer mental health in social functioning and role emotional subscales on the SF$36(\mathrm{p}=0.03$, and $\mathrm{p}=0.01$, respectively). 


\section{TELESAGE study}

TELESAGE ("Suivi A Grande Echelle d'une population de diabétiques de type 1 et de type 2 sous schéma insulinique basal bolus par la TELEmédecine" [Large-scale follow-up of a population of type 1 and type 2 diabetics under basal insulin regimen bolus by Telemedicine]) is a six-month open-label parallel-group, multicenter study, including adult patients $(n=180)$ with type 1 diabetes (>1year), on a basal-bolus insulin regimen ( $>6$ months), with $\mathrm{HbA} 1 \mathrm{c} \geq 8 \%$, conducted in approximately 100 centers in France.

These type 1 diabetic patients were randomized to usual quarterly follow-up (G1), home use of a smartphone recommending insulin doses (DiabeoTM software) with quarterly visits (G2) or use of the smartphone with short teleconsultations every 2 weeks but no visit until point end (G3). The primary objective of Télésage will be to investigate the effect of the DiabeoTM telemedicine system versus usual follow-up, with respect to improvements in the HbA1c levels (principal criterion) of diabetic patients with poorly controlled basal-bolus insulin levels $(n=696)$. The study will compare a control group (group 1 [G1]: usual follow-up) with two DiabeoTM telemedicine systems: (1) physician-assisted telemedicine (group 2 [G2]), and (2) nurse-assisted telemonitoring and teleconsultations by a diabetologist's task delegation (group 3 [G3]).

At six-month, the mean HbA1c level: $8.41 \pm 1.04 \%$ in G3 vs. $8.63 \pm 1.07 \%$ in G2 vs. $9.10 \pm 1.16 \%$ in G1 ( $p=0.0019$ for G1-G3 comparison) (Figure 3) [40]. The DiabeoTM system gave a $0.91 \%$ (0.60-1.21) improvement in HbA1c over controls and a $0.67 \%$ (0.35-0.99) reduction when used without teleconsultation. There was no difference in the frequency of hypoglycemic episodes or in medical time spent for hospital or telephone consultations. However, patients in G1 and G2 spent nearly 5h more than G3 patients attending hospital visits.

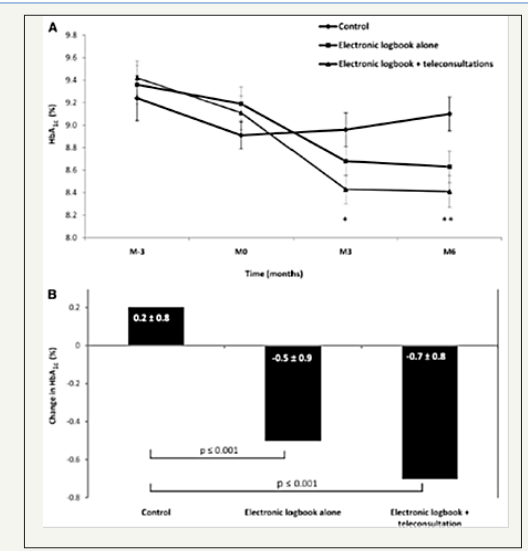

Figure 3: Efficacy of the software DiabeoTM (adapted from [40]).

\section{DIABETe project}

The DIABETe project is scheduled to experiment a telemonitoring solution for at-home monitoring of type 1 and type 2 diabetic patients. The DIABETe telemonitoring project, conducted in
Strasbourg (France), falls under the "telemedicine 2.0" category (as described above). It has been developed and designed to optimize home monitoring of diabetic patients by detecting, via a telemonitoring 2.0 platform, situations with a risk of decompensation of diabetes and its complications (e.g. MI or CHF), the latter ultimately leading to hospitalization. The AI of the DIABETe platform (MyPrediTM) automatically generates indicators of "health status" deterioration, i.e., "warning alerts" for any chronic disease worsening, particularly diabetes, its macrovascular complications and cardiovascular comorbidities (e.g. arterial hypertension, chronic heart failure). For the patient, these situations may lead to hospitalization if not treated appropriately. To our knowledge, this is one of the first projects that use $\mathrm{AI}$ in addition to ICT.

The platform comprises connected nonintrusive medical sensors (Figure 4), a touchscreen tablet connected by Wi-Fi, and a router or $3 \mathrm{G} / 4 \mathrm{G}$, rendering it possible to interact with the patient and provide education on treatment, diet, and lifestyle.

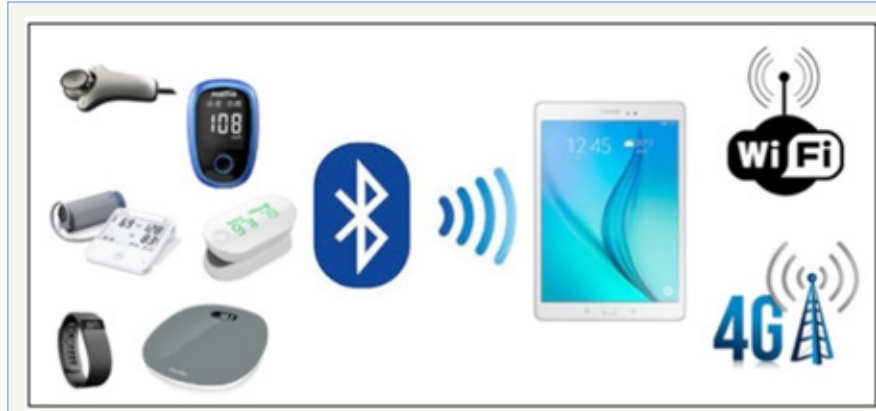

Figure 4:DIABETe's connected non-intrusive medical sensors.
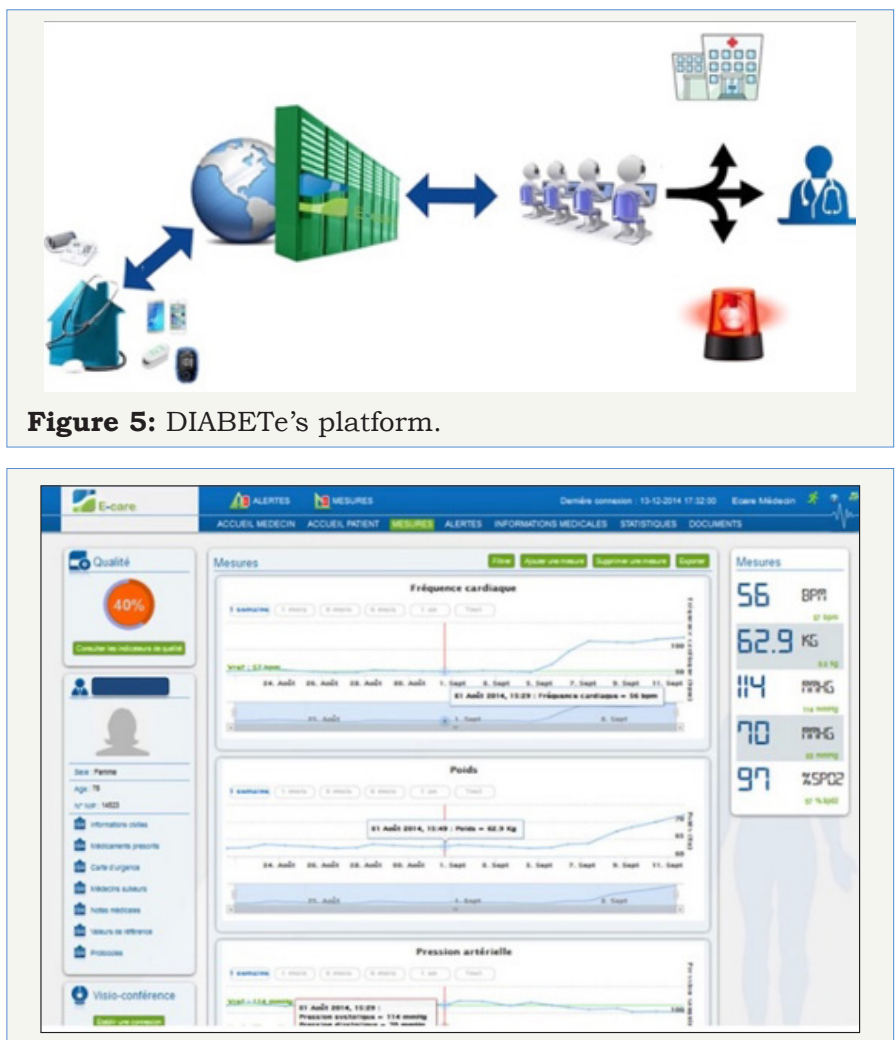

Figure 6: DIABETe's Internet portal. 
The system (Figure 5) involves a server that hosts the patient's data and a secure internet portal to which the patient and hospitaland non-hospital-based healthcare professionals can connect (Figure 6).

DIABETe is based on a smart system comprising an inference engine and a medical ontology for personalized synchronous or asynchronous analysis of data specific to each patient and, if necessary, the sending of an AI-generated alert (MyPrediTM).

DIABETe is run by a group bringing together the Strasbourg University Hospital (Hôpitaux Universitaires de Strasbourg), East Regional Health Agency (Agence Régionale de Santé du Grand Est), Bas-Rhin branch of France's National Health Insurance (Caisse Primaire d'Assurance du Bas-Rhin), and Predimed Technology start-up. This project is likely allowing an in-depth study to be carried out designed to improve diagnosis by machine learning and detect abnormalities in diabetic patients at an early time point.

The telemonitoring platform used in DIABETe was first validated in a monocentric study conducted in the Strasbourg University Hospital, carried out as part of the E-Care project, primarily focused on the problem of CHF. Between February 2014 and April 2015 175 patients (mean age of 72 years) were included into the E-care project, $30 \%$ of these patients suffered from type 2 diabetes. During this period, the telemonitoring platform was used on a daily basis by patients and healthcare professionals, according to a defined protocol of use specific to each patient. During the study, 1,500 measurements were taken, generating 700 alerts in 68 patients. 107 subjects (61.1\%) had no alerts upon follow-up. Analysis of the warning alerts in the 68 other patients showed that MyPrediTM detected any worsening of the "patient's health", with a sensitivity, specificity, as well as positive and negative predictive values of: $100 \%, 30 \%, 89 \%$ and $100 \%$, respectively. In this experimentation, both the healthcare professionals and patients, even the frailest, used the E-care system without difficulty until the end of the study.

The patients included in the DIABETe project seeks to detect the were type 1 and type 2 diabetic patients $(n=100)$ with:

A. A "very high cardiovascular risk", when presenting a personal history of myocardial infarction or stroke, limb amputation or cardiomyopathy;

B. An "intensive" insulin therapy, with at least three injections per day or pump administration, while offering them a personalized follow-up and education about their illness and its management. To date, several patients have been included. The results of this project are expected in late 2019- early 2020.

The DIABETe project is based on an intelligent platform that likely assists healthcare professionals by automatically processing the information obtained from nonintrusive medical sensors (BG meter, BP monitor, actimeter, connected scale, etc.) as well as the subjective information provided by the patient himself (questionnaires) and his/her behavior (compliance), enabling it to detect and report, at an early time, these situations at risk of hospitalization. Patient-and situation-adapted therapeutic education tools will be made available to the individual, and communication with the subject will likely occur via a touch pad. Alerts indicating a deterioration of the patient's condition will be generated by AI (new software version of MyPrediTM adapted for the management of diabetes) and transmitted to the health professionals in charge of the patient. The healthcare professional can thus anticipate the decompensation and initiate appropriate measures outside the emergency setting. An intermediate analysis is planned after the first 30 patients, possibly to set up a coordination cell with a nurse, as part of a delegation of tasks, as in TIM-HF2. Medical data can likewise be shared among health professionals, being part of a city-hospital network. Ultimately, an improvement in the patients' quality of life is to be expected.

DIABETe does not compete with DiabeoTM or other expert systems aimed at optimizing the glycemic balance, which is per se the main objective of diabetes management. The DIABETe project focuses on the "global" management of diabetic patients through the detection of situations at risk of hospitalization: infection, cardiac decompensation, diabetic foot, as well as hypoglycemia and hyperglycemia episodes, potentially leading to hospitalizations. Regarding the remote monitoring platform used in DIABETe, an integration of or interfacing with expert systems such as DiabeoTM appears possible.

\section{Perspectives Regarding New Developments in Telemed- icine}

In the future, telemedicine projects will have to address some of today's medical issues (challenge for "tomorrow telemedicine"). Thus, the new solutions of telemedicine have to take into account the coexistence in the same individual of numerous chronic pathologies (e.g. diabetes, CHF, chronic obstructive pulmonary disease, chronic renal failure, etc.) and comorbidities (high BP, dyslipidemia, etc.). They have to offer complete and "global" management, including both social and medical dimensions. They have to resolve the specificities of elderly patients: no appetite for new technologies and new uses and their main problems (e.g. falls, malnutrition, mild cognitive impairment, etc.).

In this setting, the new developments in telemedicine have also to resolve the multiplicity of health professionals working with the same patient, the multiplicity of medical organizations (e.g. with or without human resources, telemedical center, etc). Today, the logistical obstacles to the implementation of telehealth are significant, as many health systems are not yet designed to integrate these technologies into existing information systems. It is therefore necessary to plan now for an interfacing of computer systems and the integration of future telemedicine solutions.

Considering the current problems of access to healthcare professionals, the new telemedicine solutions must be able to structure the patients' care pathways, a major medical topic that should interest our governments and authorities. Likewise, the E-care and DIABETe projects provide a means for healthcare professionals to exchange with each other, thereby facilitating patient access to medical resources. In this context, future research must also focus on the accessibility and practicality of telemedicine interventions. 
Importantly, reimbursement remains a major concern and a barrier ("glass ceiling"). In fact, the healthcare delivered by telehealth is not covered by traditional fee-for-service payment models (e.g., in France, where all diabetic patients benefit from an integral treatment of their health expenses). growth of value-based payment models may, however, provide incentives to implement telehealth as a strategy to provide high-quality, cost-effective, and coordinated care. At country levels, variations in practice laws,

Table 2: Potential parameters to be evaluated in a telemedicine project for chronic disease management.

\begin{tabular}{|c|}
\hline Overall Mortality \\
\hline Specific mortality of the considered chronic disease \\
\hline Number of hospitalization for the considered chronic disease \\
\hline Number of re-hospitalization for the considered chronic disease \\
\hline Number of hospitalization days \\
\hline Heal \\
\hline Management costs for the considered chronic disease number of days off \\
work \\
\hline Quality of life
\end{tabular}

\section{Conclusion}

This short pragmatic narrative review supports the efficacy of telemonitoring diabetic patients with type 1 and type 2. Several studies on diabetes telemonitoring, using diverse technologies and transmitting different clinical, medical and behavioral data were found. Significant impacts were observed namely at the behavioral clinical and structural levels. Minimal technical problems and costeffectiveness analyses were reported.

Close management of type 1 and type 2 diabetic patients through telemonitoring showed: improvements in control of BG level, significant reduction in $\mathrm{HbA1c}$; better appropriation of the disease by patients; greater adherence to therapeutic and hygiene-dietary measures; positive impact on comorbidities (arterial hypertension, weight, dyslipidemia); better patient's quality of life; and at least, good receptiveness by patients and patient empowerment. Moreover, a cost-effectiveness analysis found a potential of medical economy. To date, the magnitude of its effects remains debatable, especially with the variation in patients' characteristics (e.g. background, ability for self-management, medical condition), samples selection and approach for treatment of control groups.

To date, relatively few projects and trials in diabetic patients have been run within the "telemedicine 2.0" setting, using AI, ICT and the Web 2.0. Nevertheless, this is the case of the project DIABETe. This project, as other projects listed in this review, are perfectly compatible with the care pathways being developed in chronic diseases by the authorities of industrialized countries, as: diabetes, chronic heart failure, chronic obstructive pulmonary disease.

Further investigation of telemonitoring efficacy and costeffectiveness over longer periods of time, and larger samples is needed. Assessment of the attitude of providers is also important considering their heavy workload and issues of reimbursement. restrictions on how telehealth can be delivered, and which patients should receive these services limit telemedicine's applicability as well.

Thus, to document the efficacy on the new telemedicine solutions, the future studies should integrate other objectives like potential targets to meet the needs and requirements of our Societies, as listed in Table 2.

\section{Therapeutic Education}

Hygiene-dietary and therapeutic compliance

Optimization of food and sports hygiene Patient self-management

Optimization of the care pathway for the considered chronic disease

Structuring of the care pathway for the considered chronic disease

\section{alth Costs}

City-hospital relations Information sharing among health professionals

System use by health professionals

\section{References}

1. Haw JS, Galaviz KI, Straus AN, Kowalski AJ, Magee MJ, et al. (2017) Longterm sustainability of diabetes prevention approaches: a systematic review and meta-analysis of randomized clinical trials. JAMA Intern Med 177(12): 1808-1817.

2. Nathan DM (2015) Diabetes: Advances in diagnosis and treatment. JAMA 314(10): 1052-1062.

3. Ostling S, Wyckoff J, Ciarkowski SL, Pai CW, Choe HM, et al. (2017) The relationship between diabetes mellitus and 30-day readmission rates. Clinical Diabetes and Endocrinology 3: 3.

4. Sinclair A, Dunning T, Rodriguez ML (2015) Diabetes in older people: New insights and remaining challenges. Lancet Diabetes Endocrinol 3(4): 275-285.

5. Ismail BF (2012) Clinical practice. Glycemic management of type 2 diabetes mellitus. N Engl J Med 366(14): 1319-1327.

6. Billiard A, Rohmer V, Roques MA, Joseph MG, Suraniti S, et al. (1991) Telematic transmission of computerized blood glucose profiles for IDDM patients. Diabetes Care 14(2): 130-134.

7. Marrero DG, Vandagriff JL, Kronz K, Fineberg NS, Golden MP, et al. (1995) Using telecommunication technology to manage children with diabetes: The Computer-Linked Outpatient Clinic (CLOC) study. Diabetes Educ 21(4): 313-319.

8. Ahring KK, Ahring JP, Joyce C, Farid NR (1992) Telephone modem access improves diabetes control in those with insulin-requiring diabetes. Diabetes Care 15(8): 971-975.

9. Liesenfeld B, Renner R, Neese M, Hepp KD (2000) Telemedical care reduces hypoglycemias and improves glycemic control in children and adolescents with type 1 diabetes. Diabetes Technol Ther 2(4): 561-567.

10. Bellazzi R, Larizza C, Montani S, Riva A, Stefanelli M, et al. (2002) A telemedicine support for diabetes management: The T-IDDM project. Comput Methods Programs Biomed 69(2): 147-161.

11. Biermann E, Dietrich W, Rihl J, Standl E (2002) Are there time and cost savings by using telemanagement for patients on intensified insulin therapy? A randomized, controlled trial. Comput Methods Programs Biomed 69(2): 137-146.

12. Welch G, Sokolove M, Mullin C (2003) Use of modem equipped blood glucose meter augmented with biweekly educator telephone support lowers HbA1c in type 1 diabetes. Diabetes 52(Suppl 1): A100. 
13. Chase HP, Roberts MD, Pearson JA, Oderberg AD, Garg SK, et al. (2003) Modem transmission of glucose values reduces the costs and need for clinic visits. Diabetes Care 26(5): 1475-1479.

14. Montori VM, Helgemoe PK, Guyatt GH, Dean DS, Leung TW, et al. (2004) Telecare for patients with type 1 diabetes and inadequate glycemic control: a randomized controlled trial and meta-analysis. Diabetes Care 27(5): 1088-1094.

15. Vahatalo MA, Virtamo HE, Viikari JS, Rönnemaa T (2004) Cellular phone transferred self-blood glucose monitoring: prerequisites for positive outcome. Pract Diab Int 21(5): 192-194.

16. Bergenstal RM, Anderson RL, Bina DM, Johnson ML, Davidson JL, et al (2005) Impact of modem-transferred blood glucose data on clinician work efficiency and patient glycemic control. Diabetes Technol Ther $7(2): 241-247$.

17. McMahon GT, Hu TMJ, Gomes HE, Hickson HS, Conlin PR, et al. (2005) Web-based care management in poorly controlled diabetes. Diabetes Care 28(7): 1624-1629.

18. Farmer AJ, Hayton PM, Gibson OJ, Tarassenko L, Neil A, et al. (2005) A randomized controlled trial of the effect of real-time telemedicine support on glycemic control in young adults with type 1 diabetes. Diabetes Care 28(11): 2697-2702.

19. Cho JH, Song KH, Chang SA, Moon SD, Yoo SJ, et al. (2006) Long-term effect of the Internet-based glucose monitoring system on HbA1c reduction and glucose stability: a 30-month follow-up study for diabetes management with a ubiquitous medical care system. Diabetes Care 29(12): 2625-2631.

20. Gay CL, Chapuis F, Bendelac N, Tixier F, Treppoz S, et al. (2006) Reinforced follow-up for children and adolescents with type 1 diabetes and inadequate glycemic control: a randomized controlled trial intervention via the local pharmacist and telecare. Diabetes Metab 32(2): 159-165.

21. Shea S, Weinstock RS, Starren J, Teresi J, Palmas W, et al. (2006) A randomized trial comparing telemedicine case management with usual care in older, ethnically diverse, medically underserved patients with diabetes mellitus. J Am Med Inform Assoc 13(1): 40-51.

22. Benhamou PY, Melki V, Boizel R, Perreal F, Quesada JL, et al. (2007) Oneyear efficacy and safety of web-based follow-up using cellular phone in type 1 diabetic patients under insulin pump therapy: The PumpNet study. Diabetes Metab 33(3): 220-226.

23. Cadario F, Binotti M, Brustia M, Mercandino F, Moreno G, et al. (2007) Telecare for teenagers with type 1 diabetes: A trial. Minerva Pediatr 59(4): 299-305.

24. Quinn CC, Clough SS, Minor JM, Lender D, Okafor MC, et al. (2008) WellDoc mobile diabetes management randomized controlled trial: change in clinical and behavioral outcomes and patient and physician satisfaction. Diabetes Technol Ther 10(3): 160-168.

25. McCarrier KP, Ralston JD, Hirsch IB, Lewis G, Martin DP, et al. (2009) Web-based collaborative care for type 1 diabetes: A pilot randomized trial. Diabetes Technol Ther 11(4): 211-217.

26. Ralston JD, Hirsch IB, Hoath J, Mullen M, Cheadle A, et al. (2009) Webbased collaborative care for type 2 diabetes: A pilot randomized trial. Diabetes Care 32(2): 234-239.

27. Shea S, Weinstock RS, Teresi JA, Palmas W, Starren J, et al. (2009) A randomized trial comparing telemedicine care management with usual care in older, ethnically diverse, medically underserved patients with diabetes mellitus: 5-year results of the IDEATel study. J Am Med Inform Assoc 16(4): 446-456.

28. https: / / www.legifrance.gouv.fr/eli/arrete/2017/4/25/ AFSH1711560A/jo/texte/fr

29. Andrès E, Hajjam M, Talha S, Meyer L, Jeandidier N, et al. (2018) Télémédecine dans le domaine de l'insuffisance cardiaque. État des lieux et focus sur le projet de télémédecine 2.0 E-care. Perspectives dans le domaine de la diabétologie. Médecine des Maladies Métaboliques 12(2): 224-231.

30. Puricel SG, Ruiz J (2014) Le diabète et l'ère de la télémédecine. Rev Med Suisse 10: 1246-1248.

31. Ponikowski P, Voors AA, Anker SD, Bueno H, Cleland JGF, et al. (2016) ESC Guidelines for the diagnosis and treatment of acute and chronic heart failure: The task force for the diagnosis and treatment of acute and chronic heart failure of the European society of cardiology (esc) developed with the special contribution of the heart failure association (HFA) of the esc. Eur Heart J 37(27): 2129-2200.

32. Stone R, Rao H, Sevick M, Cheng C, Hough LJ, et al. (2010) Active care management supported by home telemonitoring in Veterans with type 2 diabetes: the DiaTel randomized controlled trial. Diabetes Care 33(3): 478-484.

33. Stone R, Sevick M, Rao H, Macpherson DS, Cheng C, et al. (2012) The diabetes telemonitoring Study Extension: an exploratory randomized comparison of alternative interventions to maintain glycemic control after withdrawal of diabetes home telemonitoring. J Am Med Inform Assoc 19(6): 973-979.

34. Shane MW, Lenert L, Petersen M, Woolsey S, McAdam MC, et al. (2014) The utah remote monitoring project: improving health care one patient at a time. Diabetes Technol Ther 16(10): 653-660.

35. Nicolucci A, Cercone S, Chiriatti A, Muscas F, Gensini G (2015) on behalf of the REMOTE Study Group. A Randomized trial on home telemonitoring for the management of metabolic and cardiovascular risk in patients with type 2. diabetes. Diabetes Technology \& Therapeutics 17(8): 563570 .

36. Katalenich B, Shi L, Liu S, Shao H, McDuffie R, et al. (2015) Evaluation of a remote monitoring system for diabetes control. Clinical Therapeutics 37(6): 1216-1225.

37. Wild SH, Hanley J, Lewis SC, John AMcK, Lucy BMcC, et al. (2016) Supported telemonitoring and glycemic control in people with type 2 diabetes: the telescot diabetes pragmatic multicenter randomized controlled trial. PLoS Med 13(10): e1002098.

38. Turnin MC, Schirr BS, Martini J, Buisson JC, Taoui S, et al. (2017) Educ@ dom: Comparative study of the telemonitoring of patients with type 2 diabetes versus standard monitoring-study protocol for a randomized controlled study. Diabetology \& Metabolic Syndrome 9: 52.

39. Lindberg I, Torbjørnsen A, Söderberg S, Ribu L (2017) Telemonitoring and health counseling for self-management support of patients with type 2 diabetes: a randomized controlled trial. JMIR Diabetes 2(1): e10.

40. Charpentier G, Benhamou PY, Dardari D, Clergeot A, Franc S, et al. (2011) TeleDiab Study Group. The Diabeo software enabling individualized insulin dose adjustments combined with telemedicine support improves HbA1c in poorly controlled type 1 diabetic patients: A 6-month randomized, open-label, parallel-group, multicenter trial (TeleDiab 1 Study). Diabetes Care 34(3): 533-539.

41. Jeandidier N, Chaillous L, Franc S, Benhamou PY, Schaepelynck P, et al. (2018) DIABEO app software and telemedicine versus usual follow-up in the treatment of diabetic patients: protocol for the telesage randomized controlled trial. JMIR Res Protoc 7(4): e66.

42. Andrès E, Talha S, Jeandidier N, Meyer L, Hajjam M, et al. (2018) Telemedicine in chronic diseases: The time of maturity with telemedicine 2.0 in the setting of chronic heart failure and diabetes mellitus! Curr Res Diabetes Obes J 6(5): 1-4.

43. Koehler F, Koehler K, Deckwart O, Prescher S, Wegscheider K, et al. (2018) Efficacy of telemedical interventional management in patients with heart failure (TIM-HF2): a randomised, controlled, parallel-group, unmasked trial. Lancet 392(10152): P1047-P1057.

44. Van De Belt TH, Engelen L, Berben S, Schoonhoven L (2010) Definition of health 2.0 and medicine 2.0: A systematic review. J Med Internet Res 12(2): e18. 
45. Benyahia A, Hajjam A, Andrès E, Hajjam M, Hilaire V (2013) Including other system in E-Care telemonitoring platform. Stud Health Technol Inform 190: 115-117.

46. Rjeily CB, Badr G, El Hassani AH, Andrès E (2018) Medical data mining for heart diseases and the future of sequential mining. Machine Learning Paradigms: Advances in Data Analytics 149: 71-99.

47. Andrès E, Talha S, Hajjam M, Hajjam J, ErvéS, et al.(2018) Experimentation of 2.0 telemedicine in elderly patients with chronic heart failure: A study prospective in 175 patients. Eur J Intern Med 51: e11-e12.
48. Andrès E, Talha S, Benyahia AA, Keller O, Hajjam M, et al. (2015) e-Health: A promising solution for the optimized management of chronic diseases. Example of a national e-Health project E-care based on a e-plateform in the context of chronic heart failure. European Research in Telemedicine/ La Recherche Européenne en Télémédecine 4(3): 87-94.
Creative Commons Attribution 4.0 International License

For possible submissions Click Here

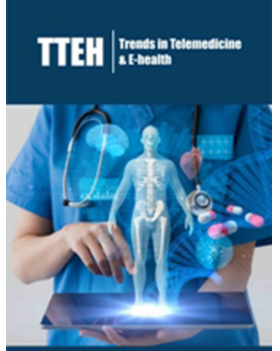

Trends in Telemedicine \& E-health

\section{Benefits of Publishing with us}

- High-level peer review and editorial services

- Freely accessible online immediately upon publication

- Authors retain the copyright to their work

- Licensing it under a Creative Commons license

- Visibility through different online platforms 